\title{
Efficacy of pleurodesis for malignant pleural effusions in breast cancer patients
}

\author{
T. Hirata*, K. Yonemori*, A. Hirakawa*, C. Shimizu*, K. Tamura*, M. Ando*, \\ N. Katsumata*, M. Tanimoto" and Y. Fujiwara*
}

ABSTRACT: This study retrospectively compared long-term outcomes between two groups of breast cancer patients with malignant pleural effusion (MPE): those receiving only systemic therapy (ST) and those receiving ST following initial pleurodesis (P-ST).

We identified 180 breast cancer patients from the National Cancer Center Hospital (Tokyo, Japan) database who had received ST and P-ST as an initial treatment for MPE between 1997 and 2008 for study inclusion. Pleural progression-free survival (PPFS) was defined as the time from ST in the ST group and from pleurodesis in the P-ST group to the first observation of pleural progression or death from any cause.

Of the 180 patients, 78 received ST and 102 received P-ST after MPE diagnosis. Median duration of follow-up was 12.7 months (range 0.9-80.1 months). Median PPFS for the ST group and the PST group was 4.1 and $\mathbf{8 . 5}$ months, respectively. The difference in PPFS between the two groups was statistically significant $(p<0.001)$ and the hazard ratio after adjusting for prognostic factors in the P-ST group relative to the ST group was 0.24 .

Our results suggest that the efficacy of P-ST may be superior to that of ST alone with respect to local control of pleural effusions in breast cancer patients.

KEYWORDS: Breast cancer, malignant pleural effusion, pleural progression-free survival, pleurodesis, systemic therapy

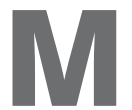
alignant pleural effusion (MPE) is a common, debilitating complication of advanced cancer. Breast cancer is the second-ranking cause of MPE and approximately $7-11 \%$ of breast cancer patients show MPE during the course of the disease [1-3]. MPE gives rise to dyspnoea, cough and chest pain, resulting in reduced activity levels and leading to an overall decrease in quality of life. An ideal treatment approach should offer long-term relief of symptoms and avoidance of the hospitalisation caused by MPE.

European Respiratory Society/American Thoracic Society guidelines state that chemotherapy with cytotoxic agents and/or hormones may be effective but that if such approaches do not relieve symptoms, local treatment options must be considered [4]. These guidelines are based on retrospective studies conducted in the 1970s and 1980s that reported that the control of pleural effusion did not significantly differ among local, systemic and combined therapies [5-7]. Systemic therapy (ST) has traditionally been the mainstay of treatment for metastatic breast cancer with or without pleurodesis. However, in an anthracycline and taxane era, it was uncertain whether ST alone or pleurodesis followed by ST (P-ST) was the more effective treatment for patients with MPE. Hence, the aim of this study was to re-evaluate these treatment strategies to determine the effect of pleurodesis on MPE in breast cancer patients. We retrospectively compared the outcomes between a group of breast cancer patients with MPE treated with ST alone and another group treated with P-ST.

\section{METHODS}

\section{Patients}

We retrieved data for 230 consecutive breast cancer patients who had been treated for MPE between 1997 and 2008 from the National Cancer Center Hospital (Tokyo, Japan) database. Inclusion in the study required: 1) documentation of MPE through a cytological examination of pleural fluid or through a pleural biopsy by video-assisted thoracic surgery (VATS); and 2) treatment with ST (i.e. chemotherapy or endocrine therapy). 24 patients who could not receive ST because of their condition due to disease progression and 26 patients who showed evidence of trapped lung were excluded. Trapped lung was defined as failed reexpansion of the lung after pleural fluid drainage, as confirmed by chest radiography. The remaining
AFFILIATIONS

*Dept of Breast and Medical

Oncology, National Cancer Center Hospital,

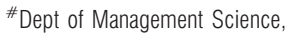
Graduate School of Engineering, Tokyo University of Science, Tokyo, and

"Dept of Hematology and Oncology, Okayama University Graduate School of Medicine, Okayama, Japan.

CORRESPONDENCE

K. Yonemori

Dept of Breast and Medical Oncology

National Cancer Center Hospital

5-1-1 Tsukiji

Chuo-ku

Tokyo 104-0045

Japan

E-mail: kyonemor@ncc.go.jp

Received:

Nov 042010

Accepted after revision:

April 292011

First published online:

May 122011 


\begin{tabular}{|c|c|c|c|}
\hline Variables & ST & P-ST & p-value ${ }^{\#}$ \\
\hline Patients & 78 & 102 & \\
\hline Age at MPE diagnosis yrs & $58(32-76)$ & $59(24-87)$ & 0.268 \\
\hline \multicolumn{4}{|l|}{ PS at MPE diagnosis } \\
\hline 0 & $6(7.7)$ & $10(9.8)$ & 0.546 \\
\hline 1 & $50(64.1)$ & $60(58.8)$ & \\
\hline 2 & $19(24.4)$ & $25(24.5)$ & \\
\hline 3 & $2(2.6)$ & $7(6.9)$ & \\
\hline 4 & $1(1.3)$ & $0(0.0)$ & \\
\hline \multicolumn{4}{|l|}{ HER2 status } \\
\hline Negative & $56(71.8)$ & $76(74.5)$ & 0.735 \\
\hline Positive & $22(28.2)$ & $26(25.5)$ & \\
\hline \multicolumn{4}{|l|}{ Hormone status } \\
\hline Negative & $20(25.6)$ & $26(25.5)$ & 1.000 \\
\hline Positive & $58(74.4)$ & $76(74.5)$ & \\
\hline \multicolumn{4}{|l|}{ Metastatic sites } \\
\hline Brain & $18(23.1)$ & 20 (19.6) & 0.586 \\
\hline Liver & $29(37.2)$ & $34(33.3)$ & 0.638 \\
\hline Lung & $59(75.6)$ & $61(59.8)$ & 0.028 \\
\hline Bone & $58(74.4)$ & $70(68.6)$ & 0.413 \\
\hline Distant lymph node & $36(46.2)$ & $35(34.3)$ & 0.125 \\
\hline Pericardium & $7(9.0)$ & $5(4.9)$ & 0.369 \\
\hline \multicolumn{4}{|l|}{$\begin{array}{l}\text { Previous chemotherapy } \\
\text { regimens } n\end{array}$} \\
\hline 0 & $55(70.5)$ & $60(58.8)$ & 0.288 \\
\hline 1 & $9(11.5)$ & $16(15.7)$ & \\
\hline$\geqslant 2$ & $14(18.0)$ & $26(25.5)$ & \\
\hline \multicolumn{4}{|l|}{$\begin{array}{l}\text { Previous endocrine } \\
\text { therapy regimens } n\end{array}$} \\
\hline 0 & $42(53.9)$ & $54(52.9)$ & 1.000 \\
\hline 1 & $13(16.7)$ & $18(17.7)$ & \\
\hline$\geqslant 2$ & $23(29.4)$ & $30(29.4)$ & \\
\hline \multicolumn{4}{|l|}{ ST after MPE diagnosis } \\
\hline Anthracycline & 19 (10.6) & 27 (26.5) & 0.863 \\
\hline Taxane & 33 (18.3) & $35(34.3)$ & 0.282 \\
\hline Endocrine therapy & $13(7.2)$ & $17(16.7)$ & 1.000 \\
\hline Fluoropyrimidine & $3(1.7)$ & $10(9.8)$ & 0.154 \\
\hline Others & $10(5.6)$ & $13(12.7)$ & 1.000 \\
\hline \multicolumn{4}{|l|}{ MPE size } \\
\hline Small-to-moderate & $53(68.0)$ & $10(9.8)$ & $<0.001$ \\
\hline Moderate-to-large & 22 (28.2) & 73 (71.6) & \\
\hline Massive & $3(3.9)$ & $19(18.6)$ & \\
\hline \multicolumn{4}{|l|}{ Need for oxygen } \\
\hline Yes & $5(6.4)$ & $44(43.1)$ & $<0.001$ \\
\hline No & 73 (93.6) & 58 (56.9) & \\
\hline
\end{tabular}

Data are presented as $n$, median (range) or $n(\%)$, unless otherwise stated. Treatment groups and sizes of malignant pleural effusion (MPE) are defined in the Methods section. ST: systemic therapy; P-ST: pleurodesis followed by ST; PS: performance status; HER: human epidermal growth factor receptor. \#: univariate analysis (t-tests and Fisher's exact tests were performed for mean differences and proportional differences between the ST and P-ST groups).

180 patients had received ST or P-ST as an initial treatment for MPE. All clinical information was collected from the patients' charts. Performance status (PS) was evaluated according to the criteria of the Eastern Cooperative Oncology Group [8]. The

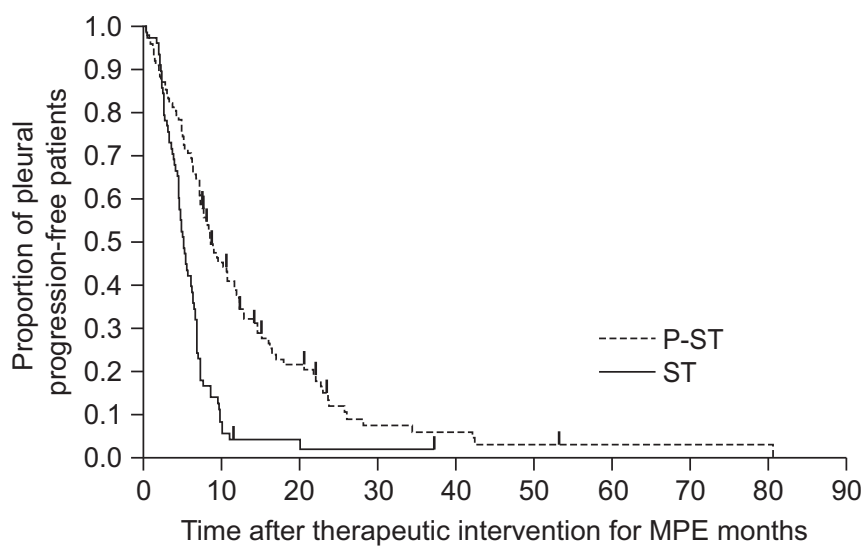

FIGURE 1. Kaplan-Meier curves of pleural progression-free survival (PPFS) in the two groups. Short vertical lines indicate censored data points. A log-rank test showed significant differences in PPFS $(p<0.001)$ between the systemic therapy (ST) alone and pleurodesis followed by ST (P-ST) groups. MPE: malignant pleural effusion.

study was approved by the Institutional Review Board of the National Cancer Center Hospital (approval number 21-013).

\section{Pleurodesis agents}

The indication for pleurodesis was determined based on a review of the following factors by a medical oncology tumour board: degree of dyspnoea, including need for oxygen; MPE size; expected response rate to ST; expected survival; and the patient's preference. The expected response rate to ST for breast cancer patients was estimated based on factors including: number of previous STs, response to previous ST (e.g. evaluation by the Response Evaluation Criteria in Solid Tumors and disease control interval), hormone status, and human epidermal growth factor receptor (HER)2 status. All the patients who received talc poudrage required pleural biopsy, and they underwent talc poudrage after VATS-assisted biopsy. Other patients underwent instillation of minocycline or OK-432. The decision to opt for instillation of either minocycline or OK-432 was made on the basis of the treating physician's preference.

Patients received a pleural sclerosant (i.e. minocycline or OK432) through a chest tube $(18 \mathrm{~F}-24 \mathrm{~F}$ in size) that had been inserted under local anaesthesia. OK-432 is a purified preparation derived from Streptococcus pyogenes type A3 that contains $0.1 \mathrm{mg}$ dried streptococci, which is equivalent to a dose of 1 Klinische Einheit (KE). OK-432 scleroses by promoting pleural fibrosis in the pleural cavity [9-11]. Intrapleural therapy was performed by instillation of either minocycline $\left(7 \mathrm{mg} \cdot \mathrm{kg}^{-1}\right.$, up to a maximum of $400 \mathrm{mg})$ or OK-432 $\left(0.2 \mathrm{KE} \cdot \mathrm{kg}^{-1}\right.$, up to a maximum of $10 \mathrm{KE}$ ) diluted in $100 \mathrm{~mL}$ saline. The tube was then clamped for $3 \mathrm{~h}$. The decision to rotate the position of the patient was based on the treating physician's preferences. Talc poudrage was performed by VATS under general anaesthesia and selective one-lung ventilation. Residual fluid was aspirated, loculi were divided when present, pleural biopsies were taken and lung re-expansion was confirmed. $5 \mathrm{~g}$ talc was insufflated. The chest tubes were removed when pleural effusion had decreased to $\leqslant 150 \mathrm{~mL} \cdot$ day $^{-1}$. 


\begin{tabular}{|c|c|c|}
\hline \multirow{2}{*}{$\begin{array}{l}\text { TABLE } 2 \\
\text { Variable }\end{array}$} & \multicolumn{2}{|c|}{$\begin{array}{l}\text { Results of multivariate Cox regression analysis of } \\
\text { pleural progression-free survival }\end{array}$} \\
\hline & HR $(95 \% \mathrm{Cl})$ & $p$-value \\
\hline \multicolumn{3}{|l|}{ Treatment group } \\
\hline ST & 1 & \\
\hline P-ST & $0.24(0.15-0.36)$ & $<0.001$ \\
\hline \multicolumn{3}{|l|}{ PS } \\
\hline $0-1$ & 1 & \\
\hline$\geqslant 2$ & $1.85(1.29-2.65)$ & 0.002 \\
\hline \multicolumn{3}{|c|}{$\begin{array}{l}\text { Previous chemotherapy } \\
\text { regimens } n\end{array}$} \\
\hline 0 & 1 & \\
\hline 1 & $1.30(0.81-2.07)$ & 0.281 \\
\hline$\geqslant 2$ & $1.64(1.09-2.47)$ & 0.017 \\
\hline \multicolumn{3}{|c|}{$\begin{array}{l}\text { Previous endocrine therapy } \\
\text { regimens } n\end{array}$} \\
\hline 0 & 1 & \\
\hline 1 & $1.34(0.83-2.15)$ & 0.226 \\
\hline$\geqslant 2$ & $1.37(0.89-2.09)$ & 0.149 \\
\hline \multicolumn{3}{|l|}{ HER2 status } \\
\hline Negative & 1 & \\
\hline Positive & $1.02(0.69-1.49)$ & 0.939 \\
\hline \multicolumn{3}{|l|}{ Hormone status } \\
\hline Negative & 1 & \\
\hline Positive & $0.65(0.41-1.03)$ & 0.064 \\
\hline \multicolumn{3}{|l|}{ MPE size } \\
\hline Small-to-moderate & 1 & \\
\hline Moderate-to-large & $1.29(0.85-1.97)$ & 0.235 \\
\hline Massive & $1.73(0.93-3.20)$ & 0.083 \\
\hline
\end{tabular}

\section{Evaluation of response and size of MPE}

Each patient underwent a pre-drainage, baseline chest radiograph. Sizes of MPE were classified into three groups on the basis of the initial chest radiographs: 1) small-to-moderate MPE was defined as occupying less than one-quarter of the hemithorax; 2) moderate-to-large was defined as occupying greater than onequarter, but less than the entire hemithorax; and 3) massive was defined as those effusions occupying the entire hemithorax. All patients received a full drainage; that is, the P-ST group underwent drainage through a chest tube and the ST group underwent therapeutic thoracentesis. The treatment response of MPE to pleurodesis was evaluated according to the following criteria: 1) complete response was defined as long-term relief of symptoms related to the effusion, with absence of fluid reaccumulation on chest radiographs until death; 2) partial response was defined as diminution of dyspnoea related to the effusion with only partial re-accumulation of fluid $(<50 \%$ of the initial radiographic evidence of fluid), with no further therapeutic thoracenteses required for the remainder of the patient's life; and 3) lack of response was defined as lack of either a complete or partial response [4].

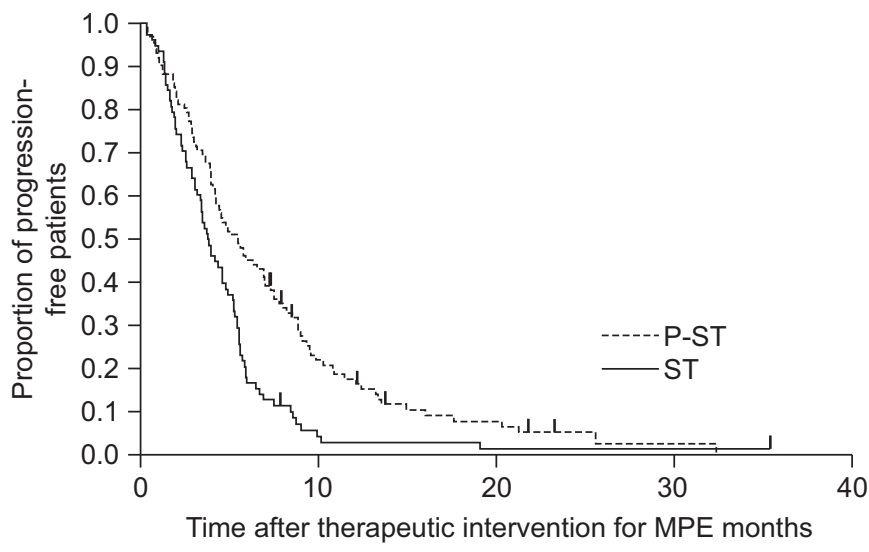

FIGURE 2. Kaplan-Meier curves of progression-free survival (PFS) in the two groups. Short vertical lines indicate censored data points. A log-rank test showed significant differences in PFS $(p<0.001)$ between the systemic therapy $(S T)$ alone and pleurodesis followed by ST (P-ST) groups. MPE: malignant pleural effusion.

\section{Statistical analysis}

The frequencies and descriptive statistics of the demographic and clinical variables from the two groups were compared using t-tests and Fisher's exact test. Pleural progression-free survival (PPFS) was defined as the time from ST in the ST group and from pleurodesis in the P-ST group to the first observation of pleural progression or to death resulting from any cause. The definition of pleural progression was the same as that for lack of response in that pleural progression occurred when neither a complete nor a partial response occurred. Progression-free survival (PFS) was defined as the time from ST in the ST group and from pleurodesis in the P-ST group to disease progression or to death due to any cause. Overall survival (OS) was defined as the time from ST in the ST group and from pleurodesis in the P-ST group to death due to any cause. PPFS, PFS and OS were estimated using the KaplanMeier method, and the survival curves were compared using the log-rank test. Multivariate Cox regression analysis was used to estimate the hazard ratio (HR), 95\% confidence interval

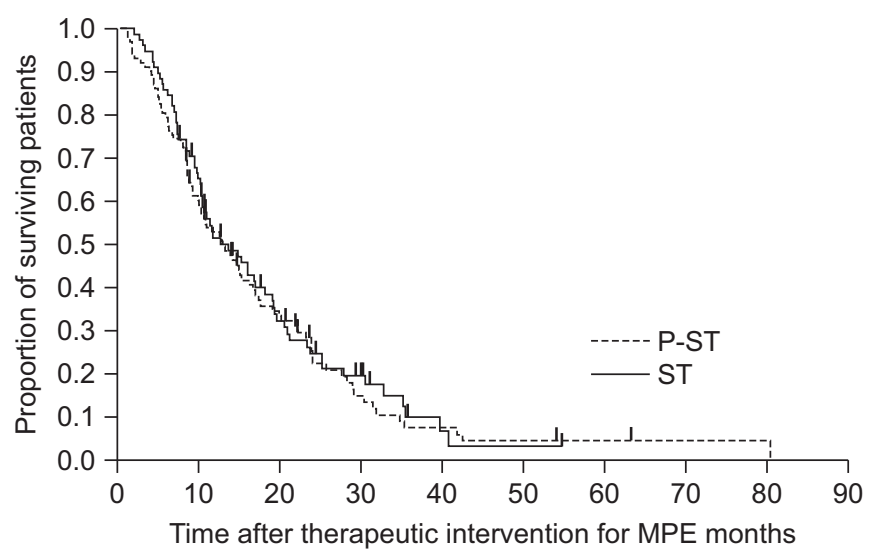

FIGURE 3. Kaplan-Meier curves of overall survival (OS) in the two groups Short vertical lines indicate censored data points. A log-rank test did not show significant differences in OS $(p=0.681)$ between the systemic therapy (ST) alone and pleurodesis followed by ST (P-ST) groups. MPE: malignant pleural effusion. 
TABLE 3 Results of multivariate Cox regression analysis of progression-free survival (PFS) and overall survival (OS)

\begin{tabular}{|c|c|c|c|c|}
\hline \multirow[t]{2}{*}{ Variable } & \multicolumn{2}{|c|}{ PFS } & \multicolumn{2}{|c|}{ os } \\
\hline & $\mathrm{HR}(95 \% \mathrm{Cl})$ & p-value & $\mathrm{HR}(95 \% \mathrm{Cl})$ & p-value \\
\hline \multicolumn{5}{|l|}{ Treatment group } \\
\hline ST & 1 & & 1 & \\
\hline P-ST & $0.43(0.29-0.64)$ & $<0.001$ & $0.91(0.60-1.38)$ & 0.658 \\
\hline$\geqslant 2$ & $1.56(1.10-2.22)$ & 0.013 & $2.74(1.85-4.06)$ & $<0.001$ \\
\hline \multicolumn{5}{|c|}{ Previous chemotherapy regimens $\mathrm{n}$} \\
\hline 0 & 1 & & 1 & \\
\hline 1 & $1.10(0.69-1.76)$ & 0.735 & $1.89(1.16-3.07)$ & 0.010 \\
\hline$\geqslant 2$ & $1.98(1.33-2.95)$ & 0.001 & $2.86(1.83-4.46)$ & $<0.001$ \\
\hline \multicolumn{5}{|c|}{ Previous endocrine therapy regimens $n$} \\
\hline \multicolumn{5}{|l|}{ HER2 status } \\
\hline Negative & 1 & & 1 & \\
\hline Positive & $1.02(0.70-1.49)$ & 0.922 & $1.10(0.74-1.64)$ & 0.641 \\
\hline \multicolumn{5}{|l|}{ Hormone status } \\
\hline Negative & 1 & & 1 & \\
\hline Positive & $0.67(0.42-1.05)$ & 0.080 & $0.49(0.30-0.81)$ & 0.005 \\
\hline \multicolumn{5}{|l|}{ MPE size } \\
\hline Small-to-moderate & 1 & & 1 & \\
\hline Moderate-to-large & $1.24(0.82-1.87)$ & 0.311 & $0.97(0.63-1.51)$ & 0.892 \\
\hline Massive & $1.43(0.78-2.61)$ & 0.243 & $1.79(0.96-3.36)$ & 0.068 \\
\hline
\end{tabular}

Treatment groups and sizes of malignant pleural effusion (MPE) are defined in the Methods section. HR: hazard ratio; ST: systemic therapy; P-ST: pleurodesis followed by ST; PS: performance status; HER: human epidermal growth factor receptor.

and effects of clinical variables. A two-sided p-value of $<0.05$ was considered to be statistically significant. All the analyses were performed using the SAS software program (version 9.1; SAS Institute Inc., Cary, NC, USA).

\begin{tabular}{lccc} 
TABLE 4 & Adverse events from & pleurodesis \\
& OK-432 & Minocycline & Talc \\
\hline Subjects & 42 & 57 & 3 \\
Leukocytopenia & $2(4.8)$ & $2(3.5)$ & $0(0.0)$ \\
Neutropenia & $1(2.4)$ & $1(1.7)$ & $1(33.3)$ \\
AST elevation & $4(9.5)$ & $0(0.0)$ & $0(0.0)$ \\
ALT elevation & $3(7.1)$ & $1(1.7)$ & $0(0.0)$ \\
Nausea/vomiting & $4(9.5)$ & $2(3.5)$ & $1(33.3)$ \\
Chest pain & $28(66.6)$ & $13(22.8)$ & $1(33.3)$ \\
Fever & $31(73.8)$ & $5(8.8)$ & $1(33.3)$ \\
Pleural infection & $2(4.8)$ & $0(0.0)$ & $0(0.0)$ \\
\hline
\end{tabular}

Data are presented as $\mathrm{n}$ or $\mathrm{n}(\%)$. Leukocytopenia was defined as a white blood cell count of $<3,900$ cells $\cdot \mu \mathrm{L}^{-1}$. Neutropenia was defined as an absolute neutrophil count of $<1,500$ cells $\cdot \mu \mathrm{L}^{-1}$. Aspartate aminotransferase (AST) elevation was defined as $>33 \mathrm{IU} \cdot \mathrm{L}^{-1}$. Alanine aminotransferase (ALT) elevation was defined as $>27 \mathrm{IU} \cdot \mathrm{L}^{-1}$

\section{RESULTS}

\section{Patient characteristics}

Of the 180 patients included in our study, 78 received ST and 102 received P-ST. The frequencies and descriptive statistics of the demographic and clinical variables in the two groups were similar except for the proportion of lung metastases, size of MPE and need for oxygen (table 1). The size of MPE and the proportion of patients requiring oxygen were larger in the P-ST group than in the ST group. In the P-ST group, the number of patients treated with minocycline, OK-432 and talc was 57 $(55.9 \%), 42(41.2 \%)$ and three $(2.9 \%)$, respectively. $55(70.5 \%)$ and $60(58.8 \%)$ patients in the ST and P-ST groups, respectively, were chemotherapy-naïve at the time of MPE diagnosis.

\section{PPFS}

The median duration of follow-up was 12.7 months (range 0.980.1 months). The median PPFS of the ST group and P-ST group was 4.1 and 8.5 months, respectively, as documented using the Kaplan-Meier curves (fig. 1). The differences between the two curves were statistically significant, as determined by the log-rank test $(p<0.001)$. The numbers of patients showing complete response and partial response of pleural effusion in the P-ST group were $45(44.1 \%)$ and 28 $(27.4 \%)$, respectively. 49 out of 78 patients in the ST group eventually received pleurodesis after progression of pleural 
effusion, and the number of patients showing complete response and partial response of pleural effusion were 20 $(40.8 \%)$ and $12(24.4 \%)$, respectively. Multivariate Cox regression analysis was performed to compare the PPFS between the two groups after adjusting for several prognostic factors (table 2). The following six variables were chosen as prognostic factors for inclusion in the Cox proportional hazard model: PS at MPE diagnosis ( 0 or 1 versus $\geqslant 2$ ); number of previous chemotherapy regimens at MPE diagnosis (0 versus 1 versus $\geqslant 2$ ); number of previous endocrine therapy regimens at MPE diagnosis ( 0 versus 1 versus $\geqslant 2$ ); HER2 status (negative versus positive); hormone status (negative versus positive); and size of MPE (small-to-moderate versus moderate-to-large versus massive). When adjusted for these prognostic factors, the PPFS of the P-ST group was significantly longer than that of the ST group (HR 0.24, 95\% CI 0.15-0.36; $\mathrm{p}<0.001$ ).

\section{PFS and OS}

The median PFS of the ST and P-ST groups was 3.8 and 5.5 months, respectively. The median OS of the ST and P-ST groups was 13.3 and 12.7 months, respectively. Figures 2 and 3 show the Kaplan-Meier curves for PFS and OS, respectively, in the two groups. The differences between the two PFS curves were statistically significant $(\mathrm{p}<0.001)$, whereas the differences between the OS curves were not $(p=0.681)$. Table 3 shows the results of the multivariate Cox regression analyses of PFS and OS. After adjusting for the prognostic factors, the PFS of the PST group was significantly longer than that of the ST group (HR $0.43,95 \%$ CI $0.29-0.64 ; \mathrm{p}<0.001$ ), but the OS was not (HR $0.91,95 \%$ CI $0.60-1.38 ; \mathrm{p}=0.658)$.

\section{Adverse events of pleurodesis}

Adverse events were assessed in all patients who received pleurodesis (table 4). The main adverse events were chest pain $(41.1 \%)$, fever $(36.2 \%)$, nausea $(6.9 \%)$ and pleural infection $(2.0 \%)$. Fever and chest pain were especially observed in the patients who received OK-432.

\section{DISCUSSION}

The present study revealed that the P-ST treatment strategy was associated with favourable control of pleural effusion compared with ST alone in breast cancer patients with MPE. The PPFS and PFS of the ST group were significantly shorter than those of the P-ST group, although the OS of the ST group was similar to that of the P-ST group. One of the main purposes of treating metastatic breast cancer is the palliation of symptoms. Prolonging the control of MPE is of clinical significance in maintaining quality of life, and based on our results, P-ST may be considered the more effective treatment strategy for controlling MPE.

Differences in chemosensitivity affect treatment strategy, including whether to perform pleurodesis for MPE. That is, P-ST is the preferred treatment for cases in which tumours show resistance to chemotherapy (e.g. nonsmall cell lung cancer) [12]. However, ST after thoracentesis is the preferred treatment for cases in which tumours appear to show chemosensitivity (e.g. small cell lung cancer and lymphoma) $[13,14]$. The response rate to ST for breast cancer is midway between that for small cell lung cancer and nonsmall cell lung cancer. Some studies have reported that control of pleural effusion was not significantly different between ST and P-ST [5-7]. Based on these studies, breast cancer patients with MPE are currently administered ST as the first choice for treatment. However, our study showed that the PPFS and PFS of the ST group were significantly shorter than those of the P-ST group. Because the pleurodesis success rate of the P-ST group was almost similar to that of the ST group after progression of pleural effusion, it seems that the timing of pleurodesis did not affect the success rates. However, because of the different conditions in each group, the success rate should be interpreted carefully. An early pleurodesis after MPE diagnosis may help in controlling MPE for a longer time because complete response and partial response define disappearance or decrease of MPE until death. MPE causes severe symptoms and decreases the quality of life. In addition to increasing limited life span, minimising discomfort due to MPE and prolonging the period of pleural effusion control are also important.

Talc has been reported as safe and efficacious for pleurodesis [15-23]. A Cochrane review reported that pleurodesis with talc provided the highest relative chance of MPE nonrecurrence when compared with bleomycin, tetracycline, mustine or chest-tube drainage alone [24]. Because talc is not commercially available in Japan, other sclerosing agents (i.e. minocycline and OK-432) have been widely used in clinical practice. Minocycline, a derivative of tetracycline, has been shown to be as effective as tetracycline in inducing pleural fibrosis in rabbits [25]. Pleurodesis with minocycline has also been reported as safe and efficacious [26] and OK-432 was shown to be more effective than other pleural sclerosing agents [9-11].

Despite yielding clinically relevant findings, our study was limited in several respects and should be carefully interpreted. First, the patient groups studied were heterogeneous in terms of sample size and characteristics. Secondly, this study was retrospective, and the results of the statistical tests were not based on randomisation but were exploratory, although the prognostic factors were adjusted using multivariate Cox regression analysis. Hence, the efficacy of pleurodesis in patients with MPE will require further evaluation using a prospective study design.

In conclusion, our study showed that the control of pleural effusion was better in breast cancer patients with MPE receiving P-ST than in those receiving ST alone. On the basis of these results, breast cancer patients with MPE who are considered to have an indication for ST should receive pleurodesis as the firstchoice therapy for long-term alleviation of symptoms.

\section{SUPPORT STATEMENT}

This work was supported by science research grants from the Ministry of Health, Labour and Welfare (Tokyo, Japan).

\section{STATEMENT OF INTEREST}

None declared.

\section{ACKNOWLEDGEMENTS}

We thank H. Tsuda (Dept of Pathology, National Cancer Center Hospital, Tokyo, Japan) for help with the pathological review.

\section{REFERENCES}

1 Apffelstaedt JP, Van Zyl JA, Muller AG. Breast cancer complicated by pleural effusion. J Surg Oncol 1995; 58: 173-175.

2 Kreisman H, Wolkove N, Finkelstein HS, et al. Breast cancer and thoracic metastases: review of 119 patients. Thorax 1983; 38: 175-179. 
3 Weichselbaum R, Marck A, Hellman S. Pathogenesis of pleural effusion in carcinoma of the breast. Int J Radiat Oncol Biol Phys 1977; 2: 963-965.

4 Antony VB, Loddenkemper R, Astoul P, et al. Management of malignant pleural effusions. Eur Respir J 2001; 18: 402-419.

5 Fentiman IS, Rubens RD, Hayward JL. Control of pleural effusions in patients with breast cancer. Cancer 1983; 52: 737-739.

6 Lees AW, Hoy W. Management of pleural effusions in breast cancer. Chest 1979; 75: 51-53.

7 Poe $\mathrm{RH}$, Qzai R, Israel RH, et al. Survival of patient with pleural involvement by breast carcinoma. Am J Clin Oncol 1983; 6: 523-527.

8 Oken MM, Creech RH, Tormey DC, et al. Toxicity and response criteria of the Eastern Cooperative Oncology Group. Am J Clin Oncol 1982; 5: 649-655.

9 Kasahara K, Shibata K, Shintani H, et al. Randomized phase II trial of OK-432 in patients with malignant pleural effusion due to nonsmall cell lung cancer. Anticancer Res 2006; 26: 1495-1499.

10 Luh KT, Yang PC, Kuo SH, et al. Comparison of OK-432 and mitomycin $C$ pleurodesis for malignant pleural effusion caused by lung cancer. A randomized trial. Cancer 1992; 69: 674-679.

11 Yoshida K, Sugiura T, Takifuji N, et al. Randomized phase II trial of three intrapleural therapy regimens for the management of malignant pleural effusion in previously untreated non-small cell lung cancer: JCOG9515. Lung Cancer 2007; 58: 362-368.

12 Livingston RB, McCracken JD, Trauth CJ, et al. Isolated pleural effusion in small cell lung carcinoma: favorable prognosis. Chest 1982; 81: 208-211.

13 Rodriguez Panadero F. Lung cancer and ipsilateral pleural effusion. Ann Oncol 1995; 6: Suppl. 3, S25-S27.

14 Weissberg D, Kaufman M, Schwecher I. Pleuroscopy in clinical evaluation and staging of lung cancer. Poumon Coeur 1981; 37: 241-243.

15 Dresler CM, Olak J, Herndon JEII, et al. Phase III intergroup study of talc poudrage vs talc slurry sclerosis for malignant pleural effusion. Chest 2005; 127: 909-915.
16 Tan C, Sedrakyan A, Browne J, et al. The evidence on the effectiveness of management for malignant pleural effusion: a systematic review. Eur J Cardiothorac Surg 2006; 29: 829-838.

17 Gasparri R, Leo F, Veronesi G, et al. Video-assisted management of malignant pleural effusion in breast carcinoma. Cancer 2006; 106: 271-276.

18 Arapis K, Caliandro R, Stern JB, et al. Thoracoscopic palliative treatment of malignant pleural effusions: results in 273 patients. Surg Endosc 2006; 20: 919-923.

19 Paschoalini MS, Vargas FS, Marchi E, et al. Prospective randomized trial of silver nitrate vs talc slurry in pleurodesis for symptomatic malignant pleural effusions. Chest 2005; 128: 684-689.

20 Marrazzo A, Noto A, Casà L, et al. Video-thoracoscopic surgical pleurodesis in the management of malignant pleural effusion: the importance of an early intervention. J Pain Symptom Manage 2005; 30: 75-79.

21 Kolschmann S, Ballin A, Gillissen A. Clinical efficacy and safety of thoracoscopic talc pleurodesis in malignant pleural effusions. Chest 2005; 128: 1431-1435.

22 Kilic D, Akay H, Kavukçu S, et al. Management of recurrent malignant pleural effusion with chemical pleurodesis. Surg Today 2005; 35: 634-638.

23 Stefani A, Natali P, Casali C, et al. Talc poudrage versus talc slurry in the treatment of malignant pleural effusion: a prospective comparative study. Eur J Cardiothorac Surg 2006; 30: 827-832.

24 Shaw P, Agarwal R. Pleurodesis for malignant pleural effusions. Cochrane Database Syst Rev 2004; 1: CD002916.

25 Light RW, Wang NS, Sassoon CS, et al. Comparison of the effectiveness of tetracycline and minocycline as pleural sclerosing agents in rabbits. Chest 1994; 106: 577-582.

26 Hatta T, Tsubuota N, Yoshimura M, et al. Intrapleural minocycline for postoperative air leakage and control of malignant pleural effusion. Kyobu Geka 1990; 43: 283-286. 